\title{
Blood-based Biomarkers of Late Recovery in Severe Traumatic Brain Injury
}

\author{
By Vanessa C. Morales BS, Kathleen F. Weaver Ph.D., \\ Caroline Schnakers Ph.D. \& Emily R. Rosario Ph.D. \\ Loyola Marymount University
}

Abstract- Background: The incidence of traumatic brain injury (TBI) in the U.S. has been estimated at 1.7 million people each year and results in $\$ 60$ billion in medical and productivity costs. A TBI can result in severe and potentially chronic cognitive and physical deficits. There has been an increased focus on the use of neurologic biomarkers for both monitoring progression and predicting clinical outcomes; however, the majority of the studies are focused on the acute phase.

Objective: In this study, the goal was to longitudinally characterize biochemical correlates of neural activity up to 2 years after injury and determine if these biomarkers correlate with functional outcomes.

Methods: Participants $(n=13)$ with severe TBI as defined by the Glasgow Coma Scale $(<8)$ who are less than 1 year from the time of injury were included in this study. Blood samples and functional outcomes (DRS and RBANS) were collected upon enrollment and at 3, 6, 12, 18, and 24 months following enrollment.

Keywords: biomarker, traumatic brain injury, outcome, cognition, chronic.

GJMR-A Classification: NLMC Code: WE 706

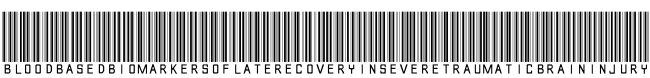

Strictly as per the compliance and regulations of:

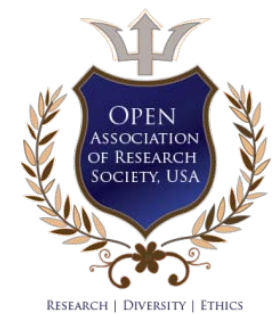

(C) 2020. Vanessa C. Morales BS, Kathleen F. Weaver Ph.D., Caroline Schnakers Ph.D. \& Emily R. Rosario Ph.D. This is a research/review paper, distributed under the terms of the Creative Commons Attribution-Noncommercial 3.0 Unported License http://creativecommons.org/licenses/by-nc/3.0/), permitting all non-commercial use, distribution, and reproduction in any medium, provided the original work is properly cited. 


\title{
Blood-based Biomarkers of Late Recovery in Severe Traumatic Brain Injury
}

\author{
Vanessa C. Morales BS ${ }^{\alpha}$, Kathleen F. Weaver Ph.D. ${ }^{\circ}$, Caroline Schnakers Ph.D. ${ }^{\rho}$ \\ \& Emily R. Rosario Ph.D. ${ }^{\omega}$
}

Abstract-Background: The incidence of traumatic brain injury (TBI) in the U.S. has been estimated at 1.7 million people each year and results in $\$ 60$ billion in medical and productivity costs. A TBI can result in severe and potentially chronic cognitive and physical deficits. There has been an increased focus on the use of neurologic biomarkers for both monitoring progression and predicting clinical outcomes; however, the majority of the studies are focused on the acute phase.

Objective: In this study, the goal was to longitudinally characterize biochemical correlates of neural activity up to 2 years after injury and determine if these biomarkers correlate with functional outcomes.

Methods: Participants $(n=13)$ with severe TBI as defined by the Glasgow Coma Scale $(<8)$ who are less than 1 year from the time of injury were included in this study. Blood samples and functional outcomes (DRS and RBANS) were collected upon enrollment and at 3, 6, 12, 18, and 24 months following enrollment. Blood-based biomarkers include Neuron-Specific Enolase (NSE), S100 calcium-binding protein B (S100B), human alpha II-spectrin breakdown product SBDP145 and 120 (SBDP145, SBDP120), and Glial fibrillary acidic protein (GFAP).

Results: A significant increase in NSE was observed over time, as well as a significant decrease in SBDP 145. NSE was also associated with an increase in cognitive abilities measured by the RBANS.

Conclusion: These results represent the first step in longitudinally characterizing blood-based biomarkers in chronic TBI and beginning to understand the relationship between blood-based biomarkers and functional outcomes.

Keywords: biomarker, traumatic brain injury, outcome, cognition, chronic.

\section{INTRODUCTION}

A traumatic brain injury (TBI) is not a onedimensional clinical entity but rather elicits a multiplicity of cellular and molecular changes in the brain(Nortje \& Menon, 2004). The result is a dynamic condition, and as such, recovery from TBI is a slow and prolonged process. Following a $\mathrm{TBI}$, individuals may experience chronic physical, cognitive, emotional and social impairments with the potential for a further decline in their daily lives years after their injury (Cuthbert et al., 2015; Pretz \& Dams-O'Connor, 2013; Pretz, Malec, \& Hammond, 2013; Sandsmark, 2016). Consequently, it is

Author a o: Loyola Marymount University, 1 LMU Drive, Los Angeles CA, USA.

Author $\rho$ w: Casa Colina Hospital and Centers for Healthcare, 255 E. Bonita Avenue Pomona, CA, USA. e-mail: erosario@casacolina.org essential to improve the prediction of a patient's prognosis specific to functional outcomes to facilitate the recovery process and improve long-term outcomes. There has been an increased focus on the use of neurologic biomarkers for both monitoring progression and predicting clinical outcomes (Agoston, ShutesDavid, \& Peskind, 2017).

To date, the majority of relevant information regarding the use of biomarkers to examine the progression of pathology caused by $\mathrm{TBI}$ is from the acute phase following injury (Yokobori et al., 2013). The most studied biomarkers of brain injury are the predominantly astrocytic-enriched proteins (i.e., S100B and glial fibrillary acidic protein, GFAP)(Agoston et al., 2017; Wang et al., 2018). Serum GFAP levels peak 20 hours after injury (Yokobori et al., 2013). GFAP has been supported as a diagnostic biomarker of acute TBI in several other large studies, including TRACK-TBI and HeadSMART in a variety of different populations. Measuring serum levels of $\mathrm{S} 100 \mathrm{~B}$ is a useful marker of brain tissue fate in TBI (Asken, Sullan, DeKosky, Jaffee, \& Bauer, 2017). This biomarker is FDA approved for TBI and is already helping clinicians and researchers today, primarily in Europe. However, its lack of specificity due to its presence outside the brain has limited its use in polytrauma; thus, this biomarker has not been widely used in North America. Clinicians have successfully implemented S100B to monitor patients to evaluate the need to perform a head CT in mild TBI patients and to detect secondary injury development, like a rise in intracranial pressure in brain-injured patients (Thelin, Johannesson, Nelson, \& Bellander, 2013).

In addition to the success of astrocytic markers for neuronal cell injury (neuronal-enriched marker neuronal specific enolase (NSE) and spectrin breakdown products (SBDPs) have also been shown to correlate with several outcome parameters (including age, Glasgow Coma Score, pupil reaction, or CT scan) obtained a few hours to 3 to 6 months after injury (Bohmer et al., 2011; Mondello et al., 2010; Vos et al., 2004). NSE has been shown to correlate with severe TBI in the acute phase in both human and animal models. SBDP 120 is produced during apoptosis, while SBDP 145 is produced due to necrosis following cell death in both human and animal models. The goal of this study was to longitudinally characterize biochemical correlates 
of neural activity and determine if these biomarkers correlate with functional outcomes.

\section{Methods}

\section{a) Participants}

Participants were eligible for the study if they were below 65 years old, had suffered a severe TBI (GCS <8) within the past year, and had a Rancho Los Amigos score greater than IV. Participants were excluded if they had a diagnosis other than TBI (i.e. spinal cord injury, other primary neurologic condition), a developmental disorder (i.e. Down syndrome), or a premorbid psychiatric conditions resulting in admission to a hospital. Eighteen participants meeting the above inclusion and exclusion criteria were enrolled in a consecutive sample for one year. Of the 18 participants enrolled, 13 completed the study in its entirety. They were between $16-61$ years of age and were between 2 to 10 months post-injury at the time of enrollment (Table 1). Written informed consent was obtained for all participants. For the participant who was 16 years of age, assent was obtained directly from the participant, as well as written informed consent from the mother.

\section{b) Study Design}

Functional status and blood samples were collected upon enrollment in the study and again at approximately $3,6,12,18$, and 24 months following enrollment. Functional status was measured with the Disability Rating Scale (DRS) and Repeatable Battery for the Assessment of Neuropsychological Status (RBANS)(Randolph, Tierney, Mohr, \& Chase, 1998; Rappaport, Hall, Hopkins, Belleza, \& Cope, 1982). A quantitative sandwich enzyme immunoassay (ELISA) technique was used to analyze serum levels of NSE (R\&D Systems), S100B (Millipore), SBDP145 (MyBioSource), SBDP120 (MyBioSource), and GFAP (Millipore). Samples for each participant were run in duplicate on the same plate. Protein levels were determined based on the standard curve using the average of the duplicate values. All research was done in accordance with required ethical standards for human subject's research.

\section{c) Data Analysis}

Blood-based biomarkers regressed against time since injury, RBANS, and the DRS using linear mixed-effects (LME) analyses customized for longitudinal data (Bernal-Rusiel, Greve, et al. 2012). The longitudinal LME analysis has the advantages of properly handling covariance among repeated measures as well as differences across patients in a number of repeated measures and time intervals between them. Each LME model also controlled for time since injury and severity of the injury.

\section{Results}

Using protein-specific sandwich ELISAs, we were able to detect levels of NSE $(1.6-11.75 \mathrm{ng} / \mathrm{ml})$, SBDP $145(0.38-11.0 \mathrm{ng} / \mathrm{ml})$, and S100B $(0.46-420.0$ $\mathrm{pg} / \mathrm{ml}$ ) in serum from chronic TBI patients over time. GFAP was only detected in 4 participants with levels from 0.12 to $6.38 \mathrm{ng} / \mathrm{ml}$. SBDP 120 was detected in 2 patients with a range from 0.33 to $7.58 \mathrm{ng} / \mathrm{ml}$. Due to the low levels of detection of these blood-based biomarkers, they were not included in further analysis.

Initial exploration of time from injury for a sample $N=13$ participants using a LME model found a significant increase in NSE $(F=18.511, p=0.001$; Figure 1) and a significant decrease in SBDP $145(F=$ 14.623, $p=0.003$; Figure 2) over time. No significant effect was observed for S100B.

When we looked at functional status and bloodbased biomarkers a significant effect was observed between increasing NSE levels and higher levels on RBANS ( $F=18.088, p=0.002$ ). We did not observe any other relationships with RBANS or the DRS and the blood-based biomarkers.

\section{Conclusion}

In this study, we characterized five potential blood-based biomarkers for the chronic phase after TBI. Although the majority of research focuses the acute phase following brain injury, where potential biomarkers will return to baseline following the injury, we know that the effects of a brain injury are chronic and may persist for years after injury. Therefore, it is important to understand what neural markers are present in this phase and if they correlate with recovery. Data collection started at $2-10$ months following a TBI and continued for two years. Of the five blood-based biomarkers that we chose to examine in chronic TBI, only three, NSE, SBDP145, and S100B, were consistently detected in our population. We observed significant changes in NSE and SBDP 145 with time since injury. Specifically, NSE increased over time, while SBDP 145 decreased with time. When we looked more closely at when this effect was taking place, we observed that the increase in NSE appeared to occur after one year following injury. NSE was also correlated with RBANS suggesting that the increase in NSE may be related to improvement in cognitive function.

Previous research has shown that GFAP and $\mathrm{S} 100 \mathrm{~B}$, both astroglial markers, collected in the acute phase of injury correlate with injury severity, CT findings, and strongly predicted mortality and poor outcomes 3 and 6 months after injury(Metting, Wilczak, Rodiger, Schaaf, \& van der Naalt, 2012; Pelinka et al., 2004; Vos et al., 2010). Astroglial activation and gliosis in response to neurodegeneration may underlie another mechanism with which GFAP could be a useful predictor in chronic 
TBI. Interestingly, in this study, we were unable to detect GFAP in all participants suggesting that is may not be a reliable marker in chronic TBI. We did detect S100B; however, we did not observe any changes over time. Previous studies have suggested S100B may be most effective following mild brain injury (Metting et al., 2012; Mondello et al., 2012).

NSE, a glycolytic enzyme that is released into the extracellular space following neuronal injury and damage, has also been shown to be a good predictor of injury(Agoston et al., 2017; Bohmer et al., 2011). Our results show elevated NSE with time and a correlation with cognitive function. Thus, NSE in chronic TBI does not appear to be representing cell death as it does in the acute phase following injury. However, NSE is expressed in erythrocytes and thus lacks neuronal specificity, potentially limiting its ability as a predictive biomarker for TBI(Agoston et al., 2017). Calpain and Caspase SBDPs, which are markers for cell death, also appears to have potential prognostic and diagnostic utility(Mondello et al., 2010; Yokobori et al., 2013). In this study, we found that SBDP 145 decreased with time but did not correlate with functional outcomes. We were not able to reliably detect SBDP120.

Taken together, these results mark the first-time biomarkers have been evaluated repeatedly through a prolonged period in TBI patients. However, there are limitations with this study, such as the small sample size, severity level (only severe TBI patients were included), and variability in time from injury upon enrollment that limits the conclusions that can be made. These findings will also benefit from a control group to determine if the changes in these blood-based biomarkers are specific to TBI. Finally, there is the possibility that the sensitivity of the markers may be contributing to the results, for example, detection of noise versus real signal. However, based on the assay specifications, the protein levels we measured do fall in the middle of the detectable range and are not near the limits of detection in either direction (i.e., too high or too low).

Treatment of TBI as a chronic condition is complicated because the symptoms and clinical needs of patients vary significantly between individuals and evolve. Through future studies, we aim to establish patterns of brain activity (MRI, EEG, blood-based neural markers) that can be associated with specific characteristics such as age, gender, presence or absence of neurological injury, and level of performance on functional tasks. Examining pathological markers for neurodegeneration such as amyloid deposition and tau phosphorylation have been shown following TBI and chronic traumatic encephalopathy, this is an area we will expand our investigation for chronic TBI. Ultimately, we believe that these blood-based biomarkers can be used to predict patient prognosis, evaluate the efficacy of therapeutic interventions, and develop adaptable personalized treatment plans that can be optimized for individuals with neurological deficits.

\section{Author Disclosure Statement}

We certify that no party having a direct interest in the results of the research supporting this article has or will confer a benefit on us or on any organization with which we are associated AND, if applicable, we certify that all financial and material support for this research (eg, NIH or NHS grants) and work are identified in the title page of the manuscript.

\section{AcKnowledgments}

The authors would like to thank Dr. Loverso, the Casa Colina Board of Directors, and the Casa Colina Foundation for supporting this research. We would also like to thank all the clinicians and research assistants who contributed to this study including Dr. Adeel Popalzai, Dr. Diem Ha Hoang, Dr. Elizabeth Cisneros, Dr. David Patterson, Dr. Kelli McSwan, Dr. Jose Fuentes, Dr. SepehrKhonsari, Dr. Earl Thurndyke, Cathy Temple, Susie Wong-Okomoto, Laura Espinoza, Stephanie Kaplan, Melissa Bustos, Kayla Vickers, Brittney Navarro, Bonnie Scudder, and Desiree Vera.

\section{Author Contribution Statement}

ER was the $\mathrm{PI}$ for this study and oversaw all aspects from design to participant recruitment to data collection, data analysis, and writing the manuscript. VM and KW completed the ELISA kits to determine protein values, provided financial support for personnel time and contributed to the statistical analysis and critical review of the manuscript. CS completed the statistical analysis and provided critical input and revision of the manuscript.

\section{References Références Referencias}

1. Agoston, D. V., Shutes-David, A., \& Peskind, E. R. (2017). Biofluid biomarkers of traumatic brain injury. Brain Inj, 31(9), 1195-1203. doi: 10.1080/ 02699052.2017.1357836

2. Asken, B. M., Sullan, M. J., DeKosky, S. T., Jaffee, M. S., \& Bauer, R. M. (2017). Research Gaps and Controversies in Chronic Traumatic Encephalopathy: A Review. JAMA Neurol, 74(10), 1255-1262. doi: 10.1001/jamaneurol.2017.2396

3. Bohmer, A. E., Oses, J. P., Schmidt, A. P., Peron, C. S., Krebs, C. L., Oppitz, P. P., . . Stefani, M. A. (2011). Neuron-specific enolase, S100B, and glial fibrillary acidic protein levels as outcome predictors in patients with severe traumatic brain injury. Neurosurgery, 68(6), 1624-1630; discussion 16301621. doi: 10.1227/NEU.0b013e318214a81f

4. Cuthbert, J. P., Pretz, C. R., Bushnik, T., Fraser, R. T., Hart, T., Kolakowsky-Hayner, S. A., . . Sherer, M. (2015). Ten-Year Employment Patterns of 
Working Age Individuals after Moderate to Severe Traumatic Brain Injury: A National Institute on Disability and Rehabilitation Research Traumatic Brain Injury Model Systems Study. Arch Phys Med Rehabil, 96(12), 2128-2136. doi: 10.1016/ j.apmr.2015.07.020

5. Metting, Z., Wilczak, N., Rodiger, L. A., Schaaf, J. M., \& van der Naalt, J. (2012). GFAP and S100B in the acute phase of mild traumatic brain injury. Neurology, 78(18), 1428-1433. doi: 10.1212/ WNL.0b013e318253d5c7

6. Mondello, S., Jeromin, A., Buki, A., Bullock, R., Czeiter, E., Kovacs, N., . . Hayes, R. L. (2012). Glial neuronal ratio: a novel index for differentiating injury type in patients with severe traumatic brain injury. $J$ Neurotrauma, 29(6), 1096-1104. doi: 10.1089/ neu.2011.2092

7. Mondello, S., Robicsek, S. A., Gabrielli, A., Brophy, G. M., Papa, L., Tepas, J., . . Hayes, R. L. (2010). alphall-spectrin breakdown products (SBDPs): diagnosis and outcome in severe traumatic brain injury patients. J Neurotrauma, 27(7), 1203-1213. doi: 10.1089/neu.2010.1278

8. Nortje, J., \& Menon, D. K. (2004). Traumatic brain injury: physiology, mechanisms, and outcome. Curr Opin Neurol, 17(6), 711-718.

9. Pelinka, L. E., Kroepfl, A., Leixnering, M., Buchinger, W., Raabe, A., \& Redl, H. (2004). GFAP versus S100B in serum after traumatic brain injury: relationship to brain damage and outcome. J Neurotrauma, 21(11), 1553-1561. doi: 10.1089/ neu.2004.21.1553

10. Pretz, C. R., \& Dams-O'Connor, K. (2013). Longitudinal description of the glasgow outcome scale-extended for individuals in the traumatic brain injury model systems national database: a National Institute on Disability and Rehabilitation Research traumatic brain injury model systems study. Arch Phys Med Rehabil, 94(12), 2486-2493. doi: 10.1016/j.apmr.2013.06.021

11. Pretz, C. R., Malec, J. F., \& Hammond, F. M. (2013). Longitudinal description of the disability rating scale for individuals in the National Institute on Disability and Rehabilitation Research traumatic brain injury model systems national database. Arch Phys Med Rehabil, 94(12), 2478-2485. doi: 10.1016/ j.apmr.2013.06.019

12. Randolph, C., Tierney, M. C., Mohr, E., \& Chase, T. N. (1998). The Repeatable Battery for the Assessment of Neuropsychological Status (RBANS): preliminary clinical validity. J Clin Exp Neuropsychol, 20(3), 310-319. doi: 10.1076/ jcen.20.3.310.823

13. Rappaport, M., Hall, K. M., Hopkins, K., Belleza, T., \& Cope, D. N. (1982). Disability rating scale for severe head trauma: coma to community. Arch Phys Med Rehabil, 63(3), 118-123.

14. Sandsmark, D. K. (2016). Clinical Outcomes after Traumatic Brain Injury. Curr Neurol Neurosci Rep, 16(6), 52. doi: 10.1007/s11910-016-0654-5

15. Thelin, E. P., Johannesson, L., Nelson, D., \& Bellander, B. M. (2013). S100B is an important outcome predictor in traumatic brain injury. J Neurotrauma, 30(7), 519-528. doi: 10.1089/neu. 2012.2553

16. Vos, P. E., Jacobs, B., Andriessen, T. M., Lamers, K. J., Borm, G. F., Beems, T., . . Vissers, J. L. (2010). GFAP and S100B are biomarkers of traumatic brain injury: an observational cohort study. Neurology, 75(20), 1786-1793. doi: 10.1212/WNL.0b013e $3181 \mathrm{fd} 62 \mathrm{~d} 2$

17. Vos, P. E., Lamers, K. J., Hendriks, J. C., van Haaren, M., Beems, T., Zimmerman, C., . . . Verbeek, M. M. (2004). Glial and neuronal proteins in serum predict outcome after severe traumatic brain injury. Neurology, 62(8), 1303-1310.

18. Wang, K. K., Yang, Z., Zhu, T., Shi, Y., Rubenstein, R., Tyndall, J. A., \& Manley, G. T. (2018). An update on diagnostic and prognostic biomarkers for traumatic brain injury. Expert Rev Mol Diagn, 18(2), 165-180. doi: 10.1080/14737159.2018.1428089

19. Yokobori, S., Hosein, K., Burks, S., Sharma, I., Gajavelli, S., \& Bullock, R. (2013). Biomarkers for the clinical differential diagnosis in traumatic brain injury--a systematic review. CNS Neurosci Ther, 19(8), 556-565. doi: 10.1111/cns.12127 
Table 1: Demographics

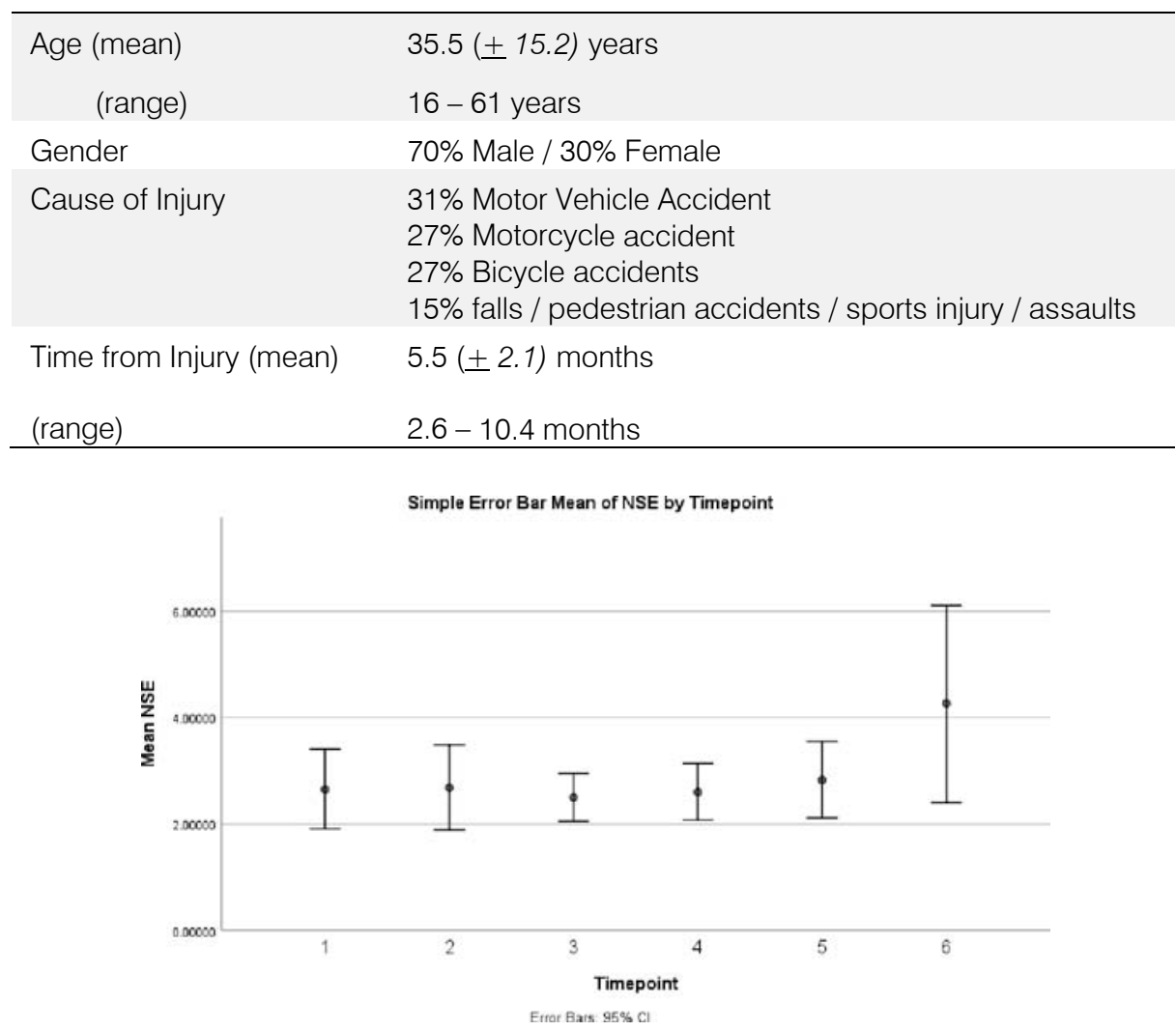

Figure 1: Data show the mean of NSE levels at 6 time points (enrollment, 3, 6, 12, 18, 24 months following enrollment) following TBI. A significant increase was observed with the increase appearing greatest after 1 year ( $F=$ $18.511, p=0.001)$.

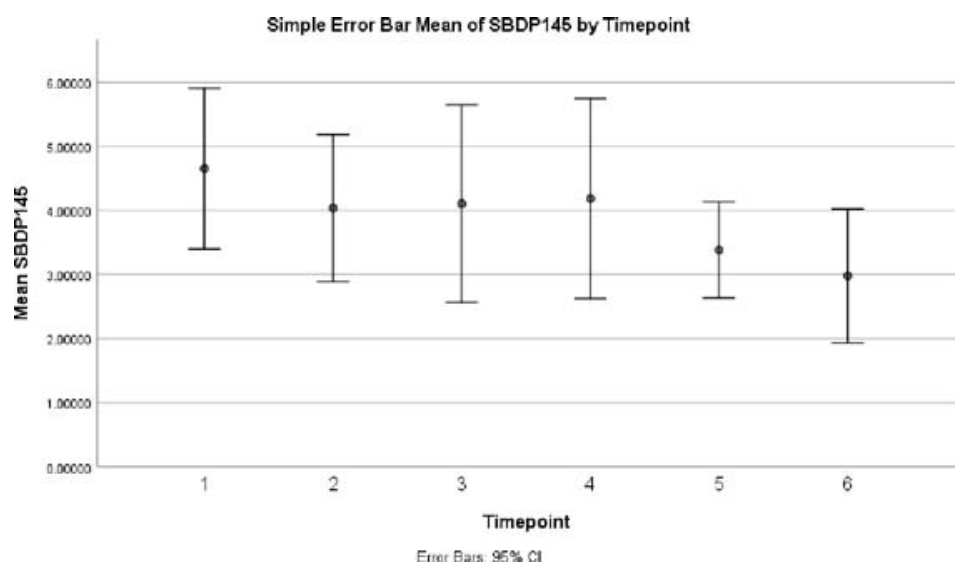

Figure 2: Data show the mean of SBDP145 levels at 6 time points (enrollment, 3, 6, 12, 18, 24 months following enrollment) following TBI. A significant decrease in SBDP 145 was observed over time ( $F=14.623, p=0.003)$. 